\title{
AUTHOR INDEX - VOL 41-2013
}

Abdullah H.Y. see Sharrad F.I. et al. (2013)

Abeyrathne A.R.N.M., Perera A.D.L.C. \& Karunaratne D.N. - Surfactant behaviour of five carbohydrate liquid crystals 41:185-194 (2013)

Abeysekera A.M., Padumadasa C. \& Mala S.M.V.D. - X-ray crystal structure analysis of 4,7-dioxononanoic acid 41: $303-307$ (2013)

Albinsson I. see Bandara T.M.W.J. et al. (2013)

Amarasiri A., Ratnayake U.N., De Silva U.K., Walpolage S. \& Siriwardene S. - Natural rubber latex-clay nanocomposite: use of montmorillonite clay as an alternative for conventional $\mathrm{CaCO}_{3}$ 41: 293-302 (2013)

Asghar S. see Naeem M. \&. Asghar S. (2013)

Athauda I.R.K. see Huffman M.A. et al. (2013)

Attanayaka A.N.P.M. \& Wijeyaratne S.C. - Corticolous lichen diversity, a potential indicator for monitoring air pollution in tropics 41:131-140 (2013)

Bandara T.M.W.J., Svensson T., Dissanayake M.A.K.L., Furlani M., Jayasundara W.J.M.J.S.R., Fernando P.S.L., Albinsson I. \& Mellander B.E. Conductivity behaviour in novel quasi-solid-state electrolyte based on polyacrylonitrile and tetrahexylammonium iodide intended for dye sensitized solar cells 41:175-184 (2013)

Banneheka B.M.S.G. see Hettiarachchi H.A.C.K. \& Banneheka B.M.S.G. (2013)

Banneheka B.M.S.G., Dhanushika M.P., Wijesuriya W. \& Herath K. - A linear programming approach to fitting an upper quadratic boundary line to natural rubber data 41:13-20 (2013)

Chandrathilaka A.M.D.S., Ileperuma O.A. \& Hettiarachchi C.V. - Spectrophotometric and pH-metric studies on Pb(II), Cd(II), Al(III) and Cu(II) complexes of paracetamol and ascorbic acid 41: 337-344 (2013)

Damunupola J.W. see Sanjeewani B.L.G. et al. (2013)

Dayawansa N.D.K. see Samarasinghe Y.M.P. \& Dayawansa N.D.K. (2013)

De Costa D.M. see Zahra A.R.F. et al. (2013)

De Costa W.A.J.M. see Zahra A.R.F. et al. (2013)

De Silva E.D. see Samarakoon K.W. et al. (2013)

De Silva L.D.G.G. see Huffman M.A. et al. (2013)

De Silva U.K. see Amarasiri A. et al. (2013)

Dhanushika M.P. see Banneheka B.M.S.G. et al. (2013)

Dharmadasa I.M., Kalyanaratne N.D.P.S.R. \& Dharmadasa R. - Effective harvesting of photons for improvement of solar energy conversion by graded bandgap multilayer solar cells 41:73-80 (2013)

Dharmadasa R. see Dharmadasa I.M. et al. (2013)

Dharmagunawardhana H.A. see Jayasingha P. et al. (2013)

Dias P. see Pathberiya H.A. \& Dias P. (2013)

Dissanayake M.A.K.L. see Bandara T.M.W.J. et al. (2013)

Ekanayake S. see Huffman M.A. et al. (2013)

Fernando K.N.R. see Mahawatte P. \& Fernando K.N.R. (2013)

Fernando M.T.R. see Sanjeewani B.L.G. et al. (2013)

Fernando M.T.R., Jayasuriya K.M.G.G., Walck J.L. \& Wijetunga A.S.T.B. - Identifying dormancy class and storage behaviour of champak (Magnolia champaca) seeds, an important tropical timber tree 41:141-146 (2013)

Fernando P.S.L. see Bandara T.M.W.J. et al. (2013)

Fernando S.C. see Vidhanaarachchi V.R.M. et al. (2013)

Furlani M. see Bandara T.M.W.J. et al. (2013)

Gopallawa D.C. \& Weerasekera K.S. - Improvement of pedestrian safety and traffic flow regulation at the railway junction, Kandy 41:259-270 (2013)

Gunasekare M.T.K. see Kottawa-Arachchi J.D. et al. (2013)

Gunatilaka A. see Premathilake R. \& Gunatilaka A. (2013)

Gunathilake K.M.D., Ratnayake R.R., Kulasooriya S.A. \& Karunaratne D.N. - Evaluation of cellulose degrading efficiency of some fungi and bacteria and their biofilms 41:155-163 (2013) 
Hamoudi A.K. see Sharrad F.I. et al. (2013)

Hasegawa H. see Huffman M.A. et al. (2013)

Hemachandra P.V. see Madurangi S.A.P. et al. (2013)

Herath K. see Banneheka B.M.S.G. et al. (2013)

Hettiarachchi C.V. see Chandrathilaka A.M.D.S. et al. (2013)

Hettiarachchi H.A.C.K. \& Banneheka B.M.S.G. - Time series regression and artificial neural network approaches for forecasting unit price of tea at Colombo auction 41:35-40 (2013)

Huffman M.A., Nahallage C.A.D., Hasegawa H., Ekanayake S., De Silva L.D.G.G. \& Athauda I.R.K. - Preliminary survey of the distribution of four potentially zoonotic parasite species among primates in Sri Lanka 41:319-326 (2013)

Ileperuma O.A. see Chandrathilaka A.M.D.S. et al. (2013)

Ishidaira H. see Wickramaarachchi T.N. et all. (2013)

Jayasingha P., Pitawala A. \& Dharmagunawardhana H.A. - Fate of urea fertilizers in sandy aquifers: laboratory and field case study from Kalpitiya, Sri Lanka 41:121-129 (2013)

Jayasinghe J.M.J.W. \& Uduwawala D.N. - Optimization of the performance of patch antennas using genetic algorithms 41:113-120 (2013)

Jayasinghe L. see Kottawa-Arachchi J.D.et al. (2013)

Jayasundara W.J.M.J.S.R. see Bandara T.M.W.J. et al. (2013)

Jayasuriya K.M.G.G. see Fernando M.T.R. et al. (2013)

Jayasuriya K.M.G.G. see Sanjeewani B.L.G. et al. (2013)

Jeon Y.J. see Samarakoon K.W. et al. (2013)

Jinasena K.D.S. \& Sonnadara D.U.J. - Computer simulation of tree development with random variations and probabilistic growth of branches 41:229-235 (2013)

Joshua W.D., Thushyanthy M. \& Nanthagoban N. - Seasonal variation of water table and groundwater quality of the karst aquifer of the Jaffna Peninsula-Sri Lanka 41:3-12 (2013)

Jude P.J. see Kannathasan S. et al. (2013)

Kalyanaratne N.D.P.S.R. see Dharmadasa I.M. et al. (2013)

Kandasamy G., Rajapakse R.P.V.J. \& Rajakaruna R.S. - Gastrointestinal and blood parasites of a free grazing flock of sheep in Kaithady farm in the Jaffna District 41:195-201 (2013)

Kannathasan S., Suthakar K., Jude P.J. \& Surendran S.N. - Potential risk areas for dengue in the Jaffna Municipal Area in Northern Sri Lanka 41:165-168 (2013)

Karunaratne D.N. see Abeyrathne A.R.N.M. et al. (2013)

Karunaratne D.N. see Gunathilake K.M.D. et al. (2013)

Karunaratne D.N. see Weerasinghe A. et al. (2013)

Karunaratne V. see Weerasinghe A. et al. (2013)

Kim E.A. see Samarakoon K.W. et al. (2013)

Kottawa-Arachchi J.D., Gunasekare M.T.K., Ranatunga M.A.B., Punyasiri P.A.N. \& Jayasinghe L. - Use of biochemical compounds in tea germplasm characterization and its implications in tea breeding in Sri Lanka 41: 309-318 (2013)

Krishnapillai N. \& Wijeratnam R.S.W. - Aspergillus rot of ripe mangoes (Mangifera indica L.) var. Ambalavi, Willard and Karuthakolumban 41:69-70 (2013)

Kulasooriya S.A. see Gunathilake K.M.D. et al. (2013)

Kulathunga D.R. \& Mahanama K.R.R. - Fingerprinting diesel and petrol fuels for adulteration in Sri Lanka 41: 287-292 (2013)

Lakmal H.H.C. see Samarakoon K.W. et al. (2013)

Lee J.H. see Samarakoon K.W. et al. (2013)

Madurangi S.A.P., Ratnasekera D., Senanayake S.G.J.N., Samarasinghe W.L.G. \& Hemachandra P.V. - Antixenosis and antibiosis effects of Oryza nivara accessions harbouring bph2 gene on brown planthopper [Nilaparvata lugens (Stal)] 41:147-154 (2013)

Magana-Arachchi D.N. \& Wanigatunge R.P. - First report of genus Chroococcidiopsis (cyanobacteria) from Sri Lanka: a potential threat to human health 41:65-68 (2013) 
Mahanama K.R.R. see Kulathunga D.R. \& Mahanama K.R.R. (2013)

Mahawatte P. \& Fernando K.N.R. - Radioactivity levels in beach sand from the West Coast of Sri Lanka - 41:279-285 (2013)

Mala S.M.V.D. see Abeysekera A.M. et al. (2013)

Mellander B.E. see Bandara T.M.W.J. et al. (2013)

Naeem M. \&. Asghar S. - A novel mutual dependence measure in structure learning 41:203-208 (2013)

Nahallage C.A.D. see Huffman M.A. et al. (2013)

Nanthagoban N. see Joshua W.D. et al. (2013)

Padumadasa C. see Abeysekera A.M. et al. (2013)

Pathberiya H.A. \& Dias P. - Modelling electricity sales in Sri Lanka and Colombo city using different statistical methods 41:41-51 (2013)

Perera A.D.L.C. see Abeyrathne A.R.N.M. et al. (2013)

Perera P.I.P. see Vidhanaarachchi V.R.M. et al. (2013)

Perera P.K., Rajapakse R.P.V.J. \& Rajakaruna R.S. - Gastrointestinal parasites of dogs in Hantana area in the Kandy District 41:81-91 (2013)

Pitawala A. see Jayasingha P. et al. (2013)

Premathilake R. \& Gunatilaka A. - Chronological framework of Asian Southwest Monsoon events and variations over the past 24,000 years in Sri Lanka and regional correlations 41:219-228 (2013)

Punyasiri P.A.N. see Kottawa-Arachchi J.D. et al. (2013)

Puvenendran S. see Weerasinghe A. et al. (2013)

Radhi R.A. see Sharrad F.I. et al. (2013)

Rajakaruna R.S. see Kandasamy G. et al. (2013)

Rajakaruna R.S. see Perera P.K. et al. (2013)

Rajapakse R.P.V.J. see Kandasamy G. et al. (2013)

Rajapakse R.P.V.J. see Perera P.K. et a. (2013)

Ranatunga M.A.B. see Kottawa-Arachchi J.D. et al. (2013)

Ratnasekera D. see Madurangi S.A.P. et al. (2013)

Ratnayake R.R. see Gunathilake K.M.D. et al. (2013)

Ratnayake U.N. see Amarasiri A. et al. (2013)

Samarakoon K.W., Lee J.H., De Silva E.D., Kim E.A., Wijesundara R.L.C., Lakmal H.H.C. \& Jeon Y.J. - Bioactivity evaluation of organic solvent extractions of Ganoderma lucidum: a Sri Lankan basidiomycete 41:249-257 (2013)

Samarasinghe W.L.G. see Madurangi S.A.P. et al. (2013)

Samarasinghe Y.M.P. \& Dayawansa N.D.K. - A remote sensing and GIS based study in assessment of the degradation risk of the Kolonnawa marsh 41: 327-335 (2013)

Sanjeewani B.L.G., Jayasuriya K.M.G.G., Fernando M.T.R. \& Damunupola J.W. - Storage and germination treatments for seeds of an ornamentally important palm, Livistona rotundifolia (Lam.) Mart. 41: 273-277 (2013)

Senanayake S.G.J.N. see Madurangi S.A.P. et al. (2013)

Sharrad F.I., Hamoudi A.K., Radhi R.A. \& Abdullah H.Y. - Inelastic electron scattering from light nuclei 41:209-217 (2013)

Siriwardene S. see Amarasiri A. et al. (2013)

Sonnadara D.U.J. see Jinasena K.D.S. \& Sonnadara D.U.J. (2013)

Sonnadara D.U.J. see Thevakaran A. \& Sonnadara D.U.J. (2013)

Subasinghe S. see Warakagoda P.S. \& Subasinghe S. (2013)

Surendran S.N. see Kannathasan S. et al. (2013)

Suthakar K. see Kannathasan S. et al. (2013)

Svensson T. see Bandara T.M.W.J. et al. (2013)

Thevakaran A. \& Sonnadara D.U.J. - Reconstruction of missing monthly temperature observations in Jaffna, Sri Lanka 41:21-28 (2013)

Thushyanthy M. see Joshua W.D. et al. (2013)

Uduwawala D.N. see Jayasinghe J.M.J.W. \& Uduwawala D.N. (2013)

Vidhanaarachchi V.R.M., Fernando S.C., Perera P.I.P. \& Weerakoon L.K. - Application of un-fertilized ovary culture to identify elite mother palms of Cocos nucifera $\mathrm{L}$. with regenerative potential 41:29-34 (2013) 
Walck J.L. see Fernando M.T.R. et al. (2013)

Walpolage S. see Amarasiri A. et al. (2013)

Wanigatunge R.P. see Magana-Arachchi D.N. \& Wanigatunge R.P. (2013)

Warakagoda P.S. \& Subasinghe S. - In vitro propagation of Pterocarpus santalinus L. (Red Sandalwood) through tissue culture 41:53-63 (2013)

Weerakoon L.K. see Vidhanaarachchi V.R.M. et al. (2013)

Weerasekera K.S. see Gopallawa D.C. \& Weerasekera K.S. (2013)

Weerasinghe A., Puvenendran S., Wickramasinghe A., Karunaratne D.N., Wijesundara S. \& Karunaratne V.) - Potent bioactivities of the endemic Annonaceae heightens its dire conservation status 41:345-350 (2013

Wickramaarachchi T.N., Ishidaira H. \& Wijayaratna T.M.N. - Variation of constituent loads and concentrations with the flow in Gin river, Sri Lanka 41:237-247 (2013)

Wickramasinghe A. see Weerasinghe A. et al. (2013)

Wijayaratna T.M.N. see Wickramaarachchi T.N. et al. (2013)

Wijeratnam R.S.W. see Krishnapillai N. \& Wijeratnam R.S.W. (2013)

Wijesundara R.L.C. see Samarakoon K.W. et al. (2013)

Wijesundara S. see Weerasinghe A. et al. (2013)

Wijesuriya W. see Banneheka B.M.S.G. et al. (2013)

Wijetunga A.S.T.B. see Fernando M.T.R. et al. (2013)

Wijeyaratne S.C. see Attanayaka A.N.P.M. \& Wijeyaratne S.C. (2013)

Zahra A.R.F., De Costa D.M. \& De Costa W.A.J.M. - Identification of differentially-expressed genes in response to salt stress in the salt-tolerant Sri Lankan rice variety At354 41:93-112 (2013) 